\title{
Discussion on Payment Application in Cross-border E-Commerce Platform from the Perspective of Blockchain
}

\author{
LIAO Qian, SHAO Mimi* \\ School of Economics \& Trade, Xinhua College of Sun Yat-sen University; Guangzhou, Guangdong, China; \\ *Corresponding Author: SHAO, Mimi
}

\begin{abstract}
Features like the distributed ledger, consensus mechanism, asymmetric encryption technology, smart contract and Token of blockchain can lower transaction cost, enhance trust between customers and merchants, as well as eliminate false payment and consumer information leakage, problems which are common in current payment of cross-border E-Commerce platform. Based on the analysis of existing scholars, this paper studied two payment models: digital cash payment based on blockchain technology and the application of blockchain in third-party payment platform. Then the paper discussed the mechanism of blockchain in cross-border e-commerce payment platform, and creatively proposed a blockchain cross-border e-commerce payment platform, serving as reference and guidance for further development of blockchain technology in cross-border payment. ${ }^{1}$
\end{abstract}

\section{Introduction}

In January 2009, 10 blockchain-based bitcoins were paid as wages to cryptographer Hal Finney. This was the first transaction completed using Bitcoin, ushering in a new era of blockchain in the field of payment.

Blockchain is regarded as a technological revolution following Internet. It is a linked data structure that combines data blocks based on time sequence while utilizing cryptography to make sure that the distributed ledger is tamper-proof and unforgeable. The data stored in the blockchain needs to be jointly maintained by the nodes of the whole network, which can effectively transfer value between nodes in the absence of trust. Compared with existing technologies, the blockchain boasts the following distinct features:

(1) Distributed ledger

The distributed ledger of blockchain stores all transaction information in the network in each block, and adds check code in the front and back blocks to make sure that blocks can supervise each other via the decentralized interlocking mechanism and support the point-to-point transmission in payment transactions.

(2) Consensus mechanism

Blockchain uses distributed consensus algorithm to form and update data, eliminating the possibility of illegal tampering at the technical level. By replacing the traditional third-party intermediary agency that guarantees credit and transaction security, it lowers transaction costs caused by credit maintenance, and saves time, labor cost and resources.
(3) Asymmetric encryption technology and smart contract

The asymmetric encryption used in blockchain encrypts transaction information through hash algorithm, and generates a pair of public and private key for each user. The public key is open to the public, while the private key is kept confidential. The private key corresponds to information encrypted by the public key, which is used to unlock the information encrypted by the private key. The private and public key can be used in information encryption and digital signature to ensure transaction security. Smart contract also uses hash algorithm to control authority and status, thus automatically updating dynamic information.

(4) Token

Token, also known as the pass certificate, is the core of blockchain. Token encourages decentralization via the distributed trust-based system. It is the driving force of blockchain technology. The existing Token wallet is similar to Alipay and WeChat wallet. The Token economy is going through an ecological exploration striving to develop from "virtual" to the "real" world. It is the trend of payment system to transform from "real" to "virtual" payment.

\section{Literature Review}

In recent years, many scholars have been doing researches on blockchain and cross-border payment. Kraus(2017) believed that blockchain digital cash is a digital cash based on blockchain technology, which uses asymmetric 
encryption of blockchain and distributed accounts to reconstruct payment transaction system. The research team of Shanghai Headquarters of the People's Bank of China (2018) proposed that blockchain can help improve the payment and clearing function of the central bank. Zou Chuanwei (2019) analyzed the application of digital cash DE/EP developed by the People's Bank of China in the field of cross-border payment, and pointed out that the issuance of digital cash will effectively expand the applicability of RMB abroad. Li Haibo (2020) proposed to solve the problem of cross-border payment through the free circulation of block chain legal digital cash. Researches by Bloomberg (2019) showed that blockchain can save time, lower transaction costs and enhance security in crossborder transactions. However, differences in regulations among countries and technology deficiency have posed constraints upon its application.

\section{Cross-border payment from the perspective of blockchain}

In 2019, the scale of cross-border E-Commerce transactions in China reached 10.5 trillion yuan, a year-onyear increase of $16.66 \%$. Cross-border E-Commerce has become one of the major forces driving trade development due to the rise of overseas E-Commerce consumption. Meanwhile, disputes and controversies caused by crossborder payment also increase with the growth of crossborder E-Commerce transactions. With the growing scale of cross-border E-Commerce, various contradictions have emerged. In 2017, there were a total of 352 litigationrelated disputes, an increase of $162.69 \%$ year-on-year. Among them, contracts and payment related disputes accounted for up to $64.31 \%$. Common disputes and problems in cross-border platform payment include transaction information tampering, misappropriation of funds, consumer information leakage, transaction credit risk, etc.

The decentralized distributed accounts of blockchain supports point-to-point transmission of greater safety and reliability, thus preventing transaction information tampering and embezzlement. Asymmetric encryption algorithm supports anonymous transaction and protects consumption information, while smart contract guarantees transaction efficiency and reduces the credit risk of transaction. The application of Token also encourages users to place orders on the platform and improves customer adhesion. The features of blockchain fit right into the technical key features of cross-border e-commerce platform, giving a full play to blockchain in cross-border payment.

\subsection{Thoughts on cross-border payment via digital cash}

The application of digital cash under blockchain in crossborder payment will be the trend in the future. Figure 1 shows the potential of digital cash in cross-border payment under blockchain. When a customer places an order from a cross-border E-Commerce platform, the block in the blockchain will record the details of the order, including information of customer, commodity and freight, and create a private and public key for customer.The blockchain account encrypts the platform payment information with the customer's public key and sends it to the customer, who can decrypt the payment information by logging in to the platform with the corresponding private key.In the payment link, the customer's signature generates a digest via hashing algorithm, then the private key is used to encrypt the digest. The signature and the text will be sent to the platform, which would decrypt the digital signature with the customer's public key after receiving the payment information and confirm that the signature is sent by the customer. Next, it uses hash function to generate a digest of the received text, and compare it with the decrypted digest. The same digest means that the received information has not been modified during transmission. The decryption of public and private key is secured without the possibility of transaction tampering. In this process,customers using digital cash for payment can complete point-to-point payment in the blockchain, that is, the digital cash of customer accounts is transmitted pointto-point to the blockchain accounts of merchants on the platform through asymmetric encryption of private and public key.The direct payment features security without third-party verification. After the goods are transported, customers confirm the receipt of goods and evaluate the purchased goods on the platform to obtain digital cash TOKEN, which can be used as marketing incentives for cross-border platforms to enhance customer adhesion and optimize shopping experience of the platform.

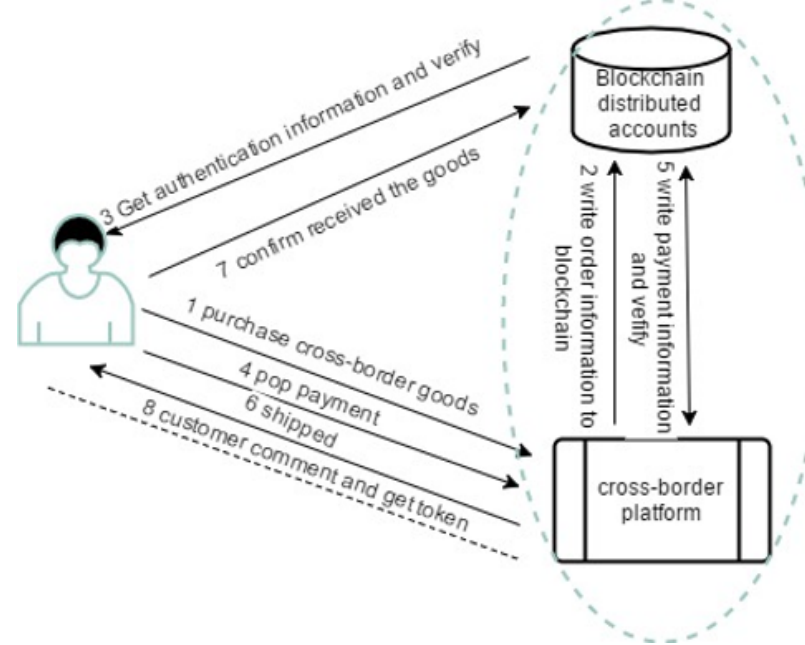

Fig1. Thoughts on Cross-border Payment Using Digital Cash under Blockchain

Bitcoin, the most popular digital cash in terms of value, is the first decentralized virtual currency based on blockchain 1.0 technology. However, the slow speed of Bitcoin blockchain in transaction and confirmation has prevented it from being fully applied in the payment field. Digital cash is developing rapidly and has a bright prospect of application in cross-border payment in the future.

\subsection{Application of blockchain in cross-border E- Commerce third-party payment platform}

The existing cross-border e-commerce platform tend to 
employ the third-party payment platform for payment. The application of blockchain technology in the third-party payment platform is shown in Figure 2. Under blockchain technology, the third-party payment platform will create two blockchain accounts for each account to cover customer and merchant. When the customer places an order on the platform, the platform will transfer the payment to the official account of the system. When the customer has no objection to receiving the goods, the official account will transfer the payment to the merchant's blockchain account.If the customer would return the goods, the refund or dispute processing system will function. In case of partial or full refund without retuning the goods, the refund system will come into play. The official account will then transfer the payment to the customer's blockchain account.

The application of blockchain in the third-party payment platform of cross-border e-commerce could eliminate false transactions, lower credit risks and transaction costs, and ensure transaction security of the payment.

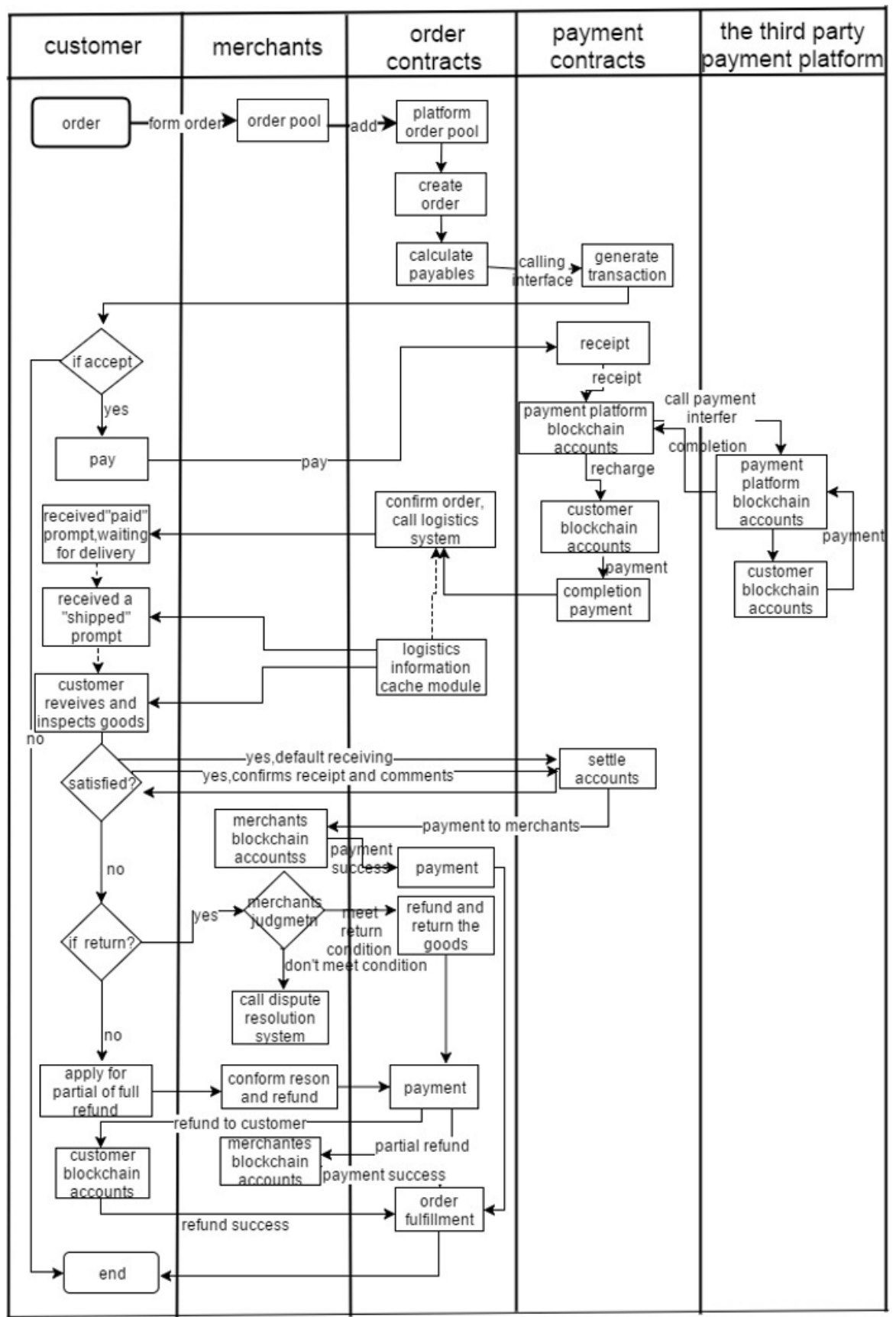

Fig2. Flow Chart of Blockchain-based Cross-border E-Commerce Platform Payment

\subsection{Payment framework of cross-border e- commerce platform from the perspective of blockchain}

The application of blockchain in the payment framework of cross-border e-commerce platform incorporates six modules: data storage, foundation support, payment system, core financial system, information platform, mobile APP or official website.

\subsubsection{Data storage module}


Data stored in the underlying blockchain is tamper-proof, allowing the front and rear blockchains to supervise each other to ensure security and reliability of transaction. The programmable smart contract is used to control and restrain the payment process in real time.

\subsubsection{Foundation support module}

The foundation support module includes the generation of public and private key for customer, supervision of consortium blockchains and regulates block information of the node.

\subsubsection{Payment system}

The payment system includes order information block, payment processing and dispute processing. The order information block contains information of customer, commodity and freight, which is stored in block account. Payment processing includes steps like payment, inquiry, cancellation, confirmation, refund and other applications, each of which corresponding to a module. Dispute processing is a crisis handling system in the payment system. Once it is called, it will start to handle disputes including agreement disputes, platform disputes and aftersales disputes, which will be handled in different modules.

\subsubsection{Core system}

The core system is a financial system in which data of realtime transaction will be recorded for subsequent reconciliation and settlement. This module has the highest security level, and will set write and read permissions.

\subsubsection{Information platform}

Information platform is mainly used for pre-sale and aftersale communication between customers and merchants.

\subsubsection{Mobile APP or official website}

Users can pay on the mobile APP or official website, which provides integrated functions including order payment, inquiry, confirmation, after-sales and other functions through the system interface.

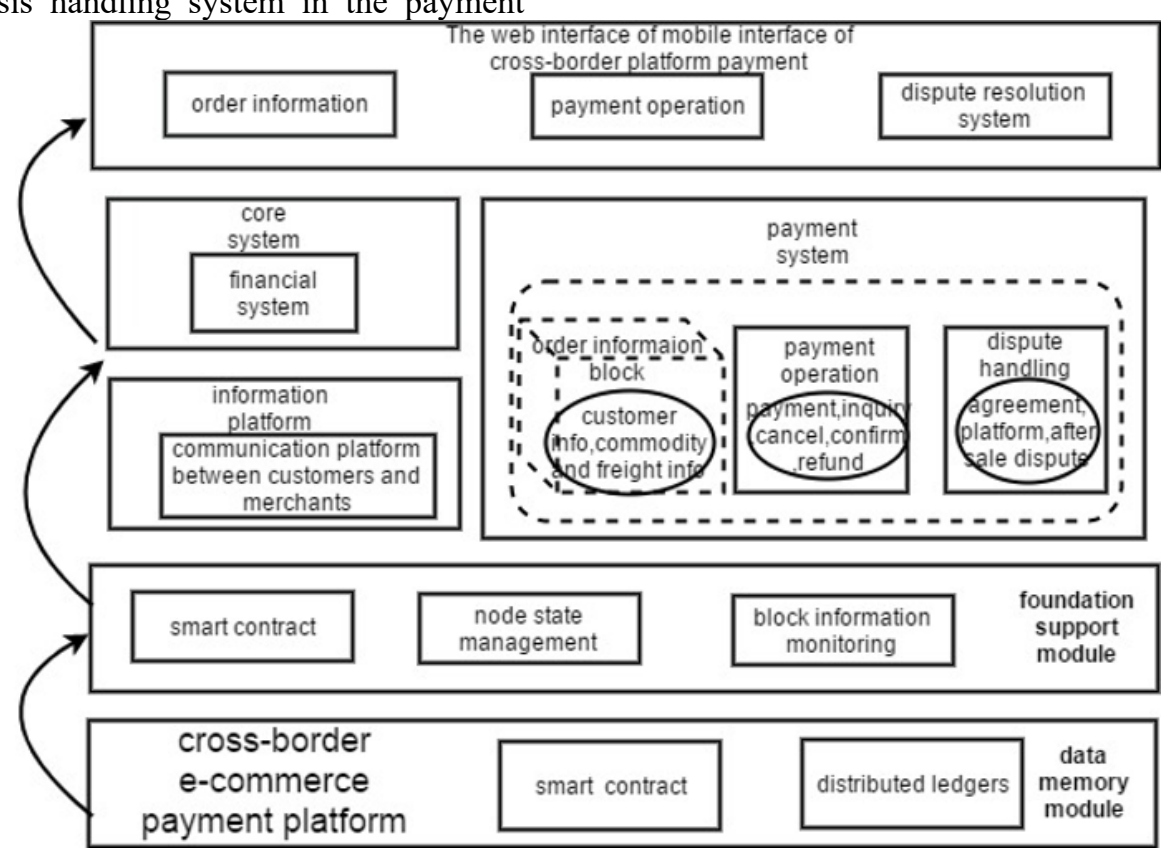

Fig3. Payment Framework of Cross-border E-Commerce Platform from the Perspective of Blockchain

\subsection{Payment model of cross-border e-commerce platform from the perspective of blockchain}

Based on the above analysis of blockchain technology and cross-border payment in combination with the overall framework of blockchain technology applied in crossborder e-commerce platform, we proposed a cross-border e-commerce payment model from the perspective of blockchain, as shown in Figure 4. This model is divided into four layers:

\subsubsection{Business layer}

The business layer is responsible for processing business logic on mobile APP or official website. The business layer is in charge of authentication, location verification, order information and payment information of customers and merchants. Users and merchants can utilize these functions on mobile APP or official website.

\subsubsection{Payment layer}

The payment layer, as a payment-based interface, is responsible for providing Paycoin-related interfaces like payment and third-party payment interfaces including exchange, Paycoin withdrawal, etc.

\subsubsection{Interface layer}

The interface layer serves as the interface for interacting with the underlying blockchain technology, including 
JSON-PRC.

\subsubsection{Blockchain}

The blockchain layer provides the platform and blockchain. In blockchain, the distributed ledger supports decentralization and point-to-point transmission, private and public key via asymmetric encryption and hash algorithm while the Merkle tree guarantees transaction security, and smart contract covers order contract and payment contract, which are mainly used to ensure the logic of major business, or the major component of data storage. As the driving force of blockchain, the pass certificate in blockchain boasts stimulating and promoting effects in the form Token.

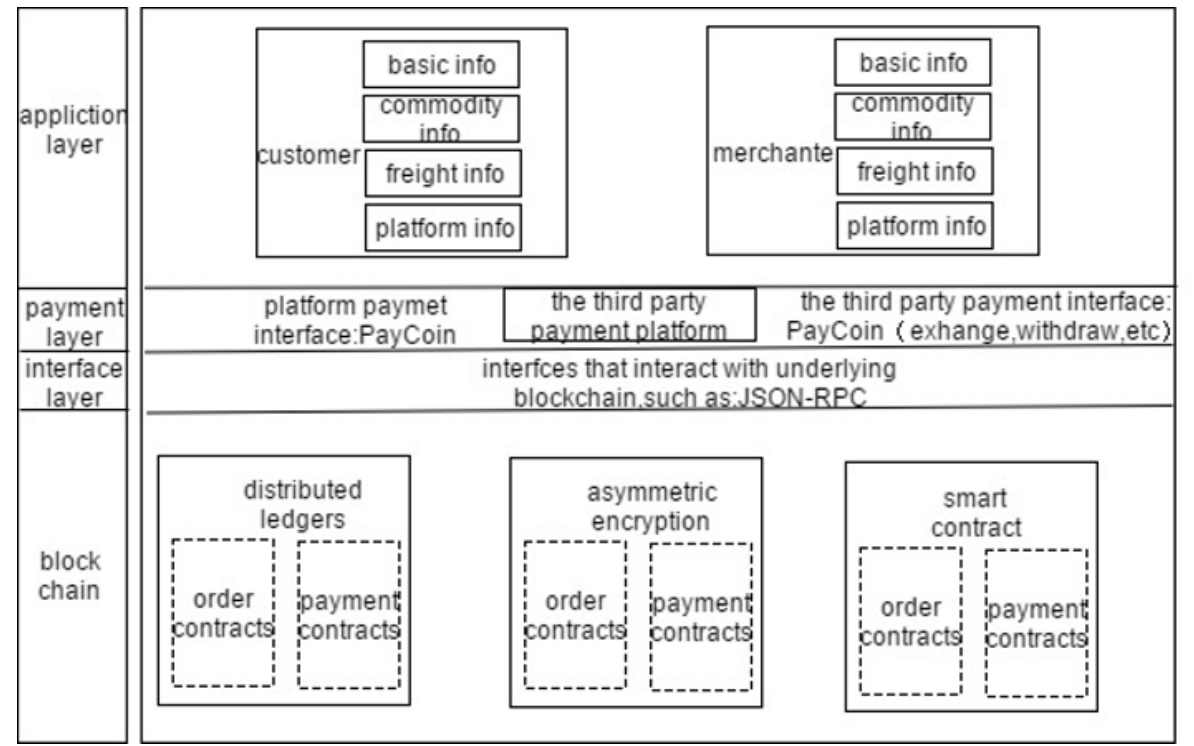

Fig4. Payment Model of Cross-border E-Commerce Platform from the Perspective of Blockchain

\section{Conclusion}

The research and achievements of this paper aim to serve as references and guidance for further development of blockchain technology in cross-border payment. "Blockchain + cross-border e-commerce" offers an opportunity of innovation for trade. Although blockchain is still in the initial stage of development with few application scenarios, and there are still problems in crossborder payment such as slow transaction and response, blockchain has a bright prospect in facilitating payment on cross-border e-commerce platform. The updated theoretical and practical development will go hand in hand to promote the application of "blockchain + cross-border e-commerce" in the future.

\section{Acknowledgment}

1 This paper is the phased achievement of "public management" construction project (F2017STSZD01), a key discipline with characteristics of Guangdong Province; and the scientific research project "blockchain + cross border e-commerce: application and future of new technology enabled e-commerce (2019KYYB09)" of Xinhua College of Sun Yat-sen University.

\section{References}

1. Li Haibo, "Research on the application of block chain legal digital currency system in cross border payment," [J]. Finance and economics, 2020 (06): 9-74.

2. Peng Bo, "Advantages, application and
Enlightenment of blockchain technology in cross border payment," [J]. Foreign trade practice, 2019 (11): 57-60.

3. Zou Chuanwei, "Blockchain and financial infrastructure -- Also on the risk and supervision of Libra project," [J]. Financial supervision research, 2019 (07): 18-33.

4. Zhang Yanbin, "Building China EU cross border ecommerce ecosystem with blockchain technology," [J]. China circulation economy, 2018,32 (02): 66-72.

5. Kraus P, "Digital Currency:Risks,Rewards and Investigative Techniques,'[D].Utica College,2017.

6. Ding Baogen, Zhao Yu, Peng Yongzhang. "The reality, limitation and policy suggestions of 'blockchain + cross border e-commerce' Reform," [J]. Contemporary economic management: 1-8.

7. Fu Yangyang, Liang Zijing. "Research on cross border e-commerce logistics traceability system of skin care products based on blockchain,"[J]. China business theory, 2018 (14): 7-9.

8. Ding Qingyang, Zhu Jianming. "B2C e-commerce platform product information traceability and anticounterfeiting model from the perspective of blockchain," [J]. China circulation economy, 2017 (12): 41-49.

9. Wang Junzhe. "Application Research on blockchain technology in cross-border e-commerce payment mode optimization,"[J]. Economic Research Guide, 2019 (10): 121-122.

10. Yang Bohan. "Application of blockchain technology to optimize cross-border e-commerce payment mode,'[J]. Contemporary economy, 2018 (14): 62-63. 
11. Wang Fei. "Blockchain technology and new ideas for promoting the development of cross-border ecommerce in China," [J]. Theory monthly, 2019 (03): 117-122.

12. Zhang Fan, Zhang Baoming. "Application of blockchain technology in cross border e-commerce," [J]. E-commerce, 2019 (03): 57-78. 